\title{
Deformation, Vibration, Buckling of Continuum Nanotorus
}

\author{
Bohua Sun \\ Department of Mechanical Engineering, Cape Peninsula University of Technology, P.O. Box 1906, Bellville 7535, \\ City of Cape Town, South Africa
}

Correspondence should be addressed to Bohua Sun, sunb@cput.ac.za

Received 12 May 2010; Accepted 25 July 2010

Academic Editor: Xuedong Bai

Copyright (c) 2010 Bohua Sun. This is an open access article distributed under the Creative Commons Attribution License, which permits unrestricted use, distribution, and reproduction in any medium, provided the original work is properly cited.

\begin{abstract}
A nanotorus is theoretically described as carbon nanotube bent into a torus (doughnut shape). Nanotori are predicted to have many unique properties, such as magnetic moments 1000 times larger than previously expected for certain specific radii. Its properties vary widely depending on radius of the torus and radius of the tube. Here, we developed a continuum model of nanotorus and obtained a closed form solutions for nanotorus deformation, vibration, and buckling and embedded in an elastic medium. The nanotorus is considered as a continuum model.
\end{abstract}

\section{Introduction}

Since its discovery, nanotube has attracted great attention because of its unusual properties [1]. Owing to its exceptional mechanical properties and low density, nanotube is an ideal material for use in nanoelectromechanical systems (NEMS), which are of great interest both for fundamental studies of mechanics at the nanoscale and for a variety of applications [2].

Carbon nanotubes are the strongest and stiffest materials yet discovered in terms of tensile strength and elastic modulus, respectively. This strength results from the covalent $\mathrm{sp}^{2}$ bonds formed between the individual carbon atoms. In 2000, a multiwalled carbon nanotube was tested to have a tensile strength of 63 gigapascals (GPa). This, for illustration, translates into the ability to endure tension of a weight equivalent to $6422 \mathrm{~kg}$ on a cable with crosssection of $1 \mathrm{~mm}^{2}$. Since carbon nanotubes have a low density for a solid of 1.3 to $1.4 \mathrm{~g} \cdot \mathrm{cm}^{-3}$, their specific strength of up to $48,000 \mathrm{kN} \cdot \mathrm{m} \cdot \mathrm{kg}^{-1}$ is the best of known materials, compared to high-carbon steel's $154 \mathrm{kN} \cdot \mathrm{m} \cdot \mathrm{kg}^{-1}$. Comparing its strength stress, unfortunately, CNTs are not nearly as strong under compression. Because of their hollow structure and high aspect ratio, they tend to undergo buckling when placed under compressive, torsion, or bending stress.

A nanotorus is theoretically described as carbon nanotube bent into a torus (doughnut shape). Nanotori are predicted to have many unique properties, such as magnetic moments 1000 times larger than previously expected for certain specific radii, or may be used as a black body whose emissivity or absorbance is almost of 1.0. Its properties vary widely depending on radius of the torus and radius of the tube. It is expected that the nanotorus has also have unique mechanical properties.

Comparing the comprehensive studies on the nanotube with cylindrical nanostructures, the nanotorus has not been well investigated [3-8].

Modelling the behaviour of the nanotorus is crucial for device design and interpretation of experimental results. Because experiments at the nanoscale are extremely difficult and atomistic modelling remains prohibitively expensive for large-sized atomic system, it has been accepted that continuum models will continue to play an essential role in the study of nanotorus as a toroidal shells. The validity of using continuum model for nanotube and graphene has been supported by both experimental results and moleculardynamics simulation; all previous investigation on nanotube has indicated that the laws of continuum mechanics are still valid to some extent even in nanoscale. The successful of continuum model to nanotube gives us a confidence to predict that continuum modelling is going to be also valid for nanotori mechanics analysis $[7,8]$.

Due to the development of nanopolymer or nanocomposites, considerable attention has turned to mechanical 


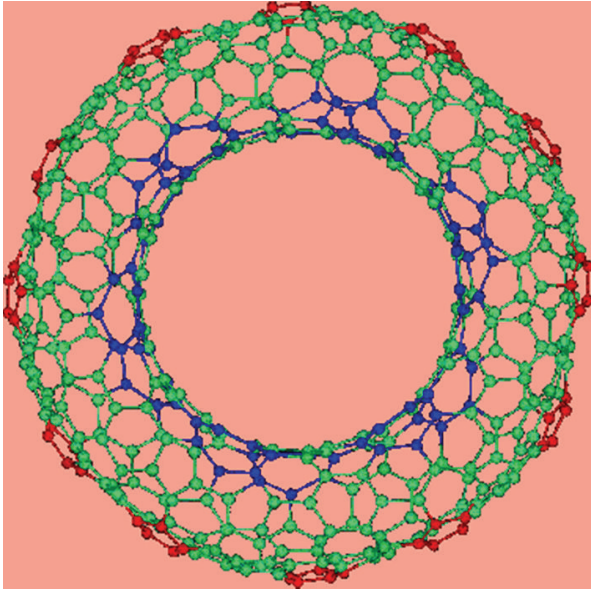

(a)

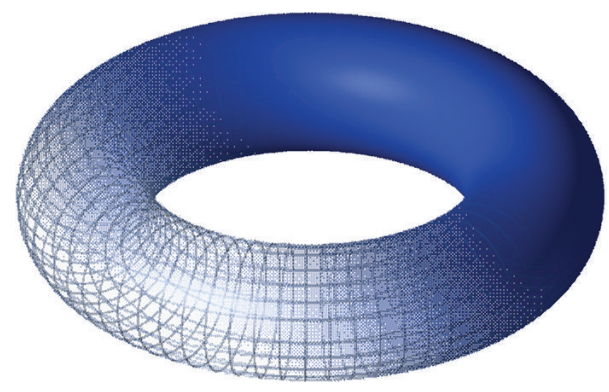

(b)

FIGURE 1: Complete nanotorus and its continuum model.

behaviour of single-walled nanotube embedded in a polymer or metal matrix. The Winkler elastic model will be adopted to study the nanotorus embedded in a elastic medium.

Although we wish to formulate the nanotorus using toroidal shell model, unfortunately, mathematically, the governing equations of toroidal shells are very complicated due to their strong variable confidents and singularity at turning point when $\sin \varphi=-1 / \alpha=-R / a$; it is very difficult to find an exact solution and numerical solution valid for whole range of $0 \leq \varphi \leq 2 \pi$, where $a$ is the radius of the toroidal shell and $R$ is the distance from the centre of the toroidal shell to the axes of the shell. The full history and discussion of toroidal shell can be found in [9].

To simplify the toroidal shell modeling, we have noticed a factor that nanotubes have been constructed with length-todiameter ratio of up to $132,000,000: 1$, which is significantly larger than any other material. It means that nanotorus's $\alpha=a / R$ is very small and delectable! Nanotorus can be considered as a very thin toroidal shell but not a solid ring.

Following our previous work in [9], after neglecting nanotorus's $\alpha=a / R$, the general toroidal equation will be reduced to a simple constant coefficient partial differential equation for slender toroidal shells, and this equation can be easily solved in closed form. As a natural extension of present works, we also demonstrated a closed-form solution for nanotorus embedded in elastic medium. In view of the difficulty in the development of molecules-dynamics

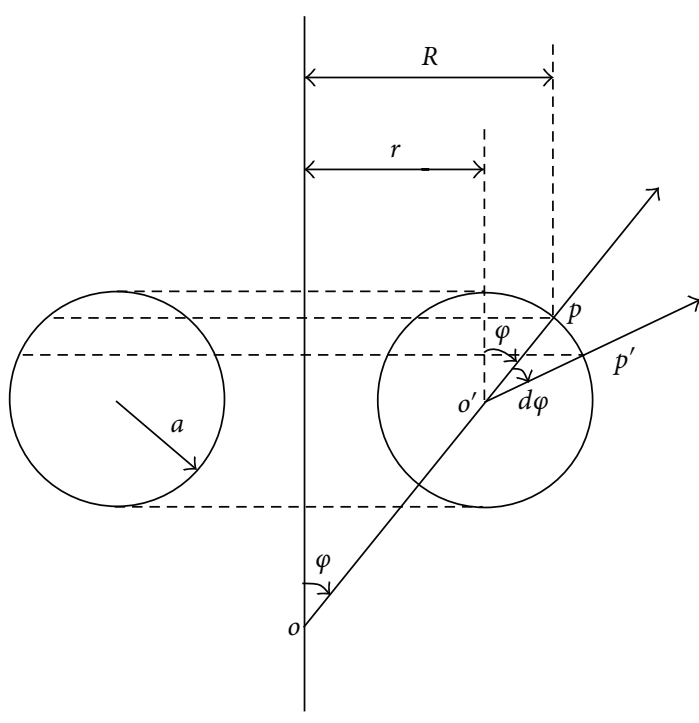

FIgURE 2: Geometrical description of nanotorus.

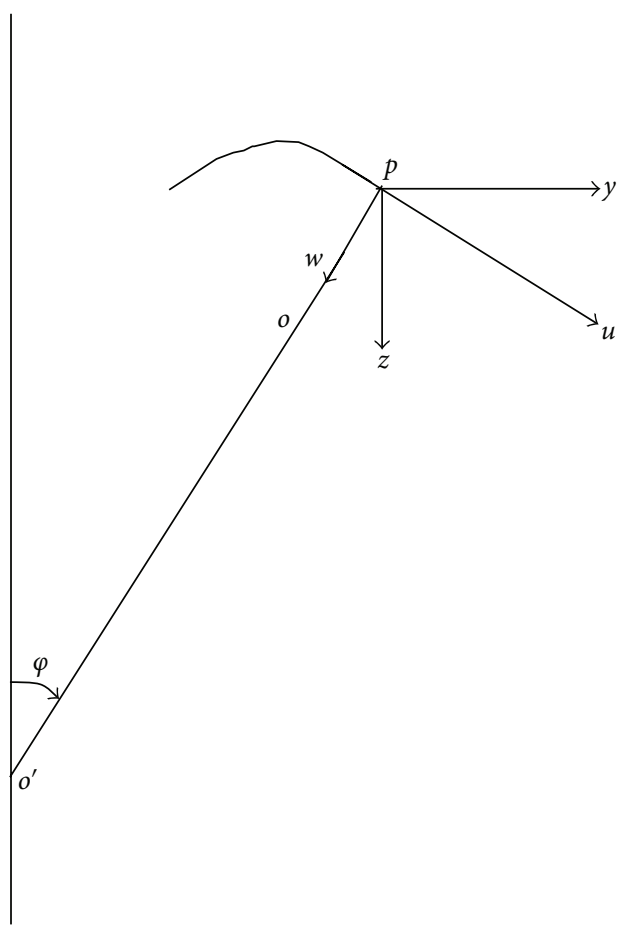

FIGURE 3: Displacement of nanotorus.

simulation, the simpler solutions obtained here are really useful for the design device.

\section{Toroidal Shell Model}

The nature of toroidal shells with circular cross-section is that one principal radius is constant and another is variable. From Figures 1, 2, 3, and 4, we have some useful relations as follows: $R_{1}=a, R_{2}=R(1+\alpha \sin \varphi) / \sin \varphi, r=R_{2} \sin \varphi=$ $R(1+\alpha \sin \varphi), \alpha=a / R$; in which $\varphi$ is the angle between the 


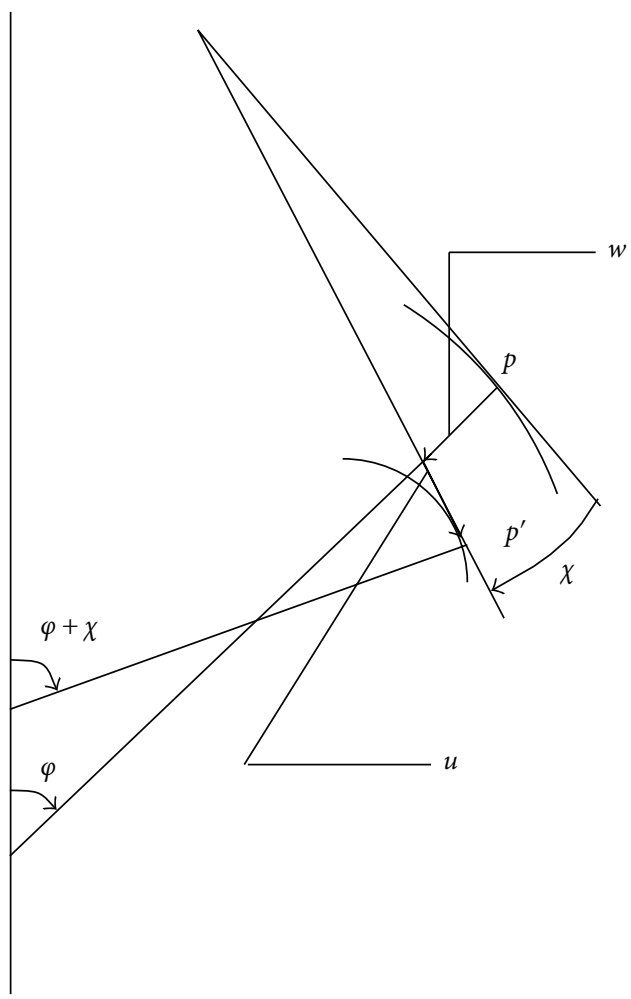

FIGURE 4: Rotation of normal of nanotorus.

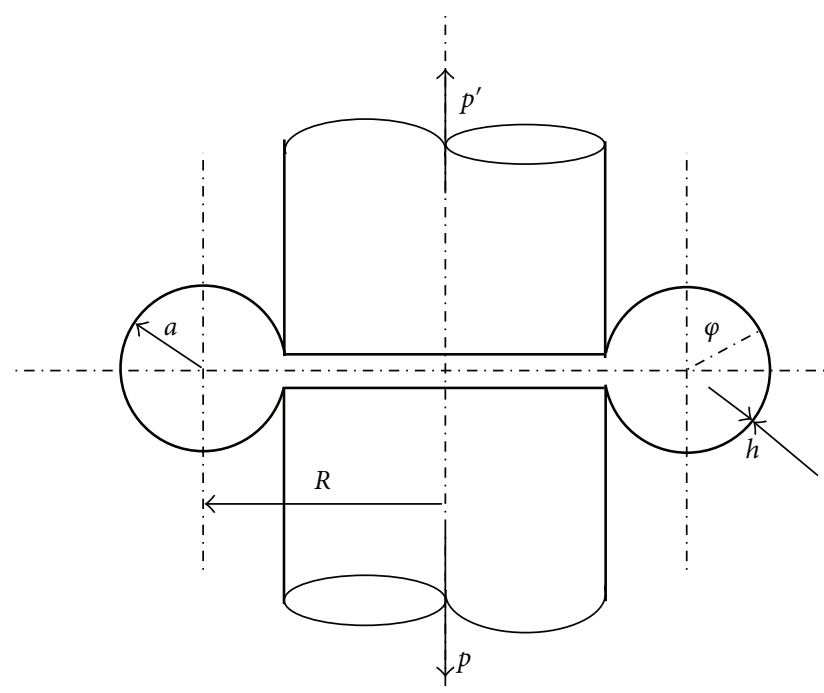

FIgURE 5: The nanotorus under concentrated loading. The separation displacement can be found as $\delta_{\text {Sun }}=\left(\pi^{2} / 6\right)\left(P a^{3} / R D\right)=$ $1.644934\left(P a^{3} / R D\right)$, where bending stiffness $D=E h^{3} /\left[12\left(1-\mu^{2}\right)\right]$, $E$ is Young modulus of the nanotori.

symmetric axes and the normal of the toroidal shell, $R_{1}$ and $R_{2}$ are the radii of curvature of the principal directions of the middle surface, $a$ is the radius of the toroidal shell, and $R$ is the distance from the centre of the toroidal shell to the axes of the shell. It is important to point out that the second radius $R_{2}=(R / \sin \varphi)+a=R(1+\alpha \sin \varphi) / \sin \varphi$ is function of $\varphi$ and has two singularity points because it become infinite at points of $\varphi=\pi / 2$ and $3 \pi / 2$. This singularity is the real source of difficulty of finding exact solution. The novel methods to find exact solution of any toroidal shells are generally taking a strategy in introducing a transformation to remove the singularity.

Internal resultant forces $T_{1}, T_{2}$ and moments $M_{1}, M_{2}$ of the shells as follows

$$
\begin{aligned}
T_{1} & =K\left[\frac{1}{a}\left(\frac{d u}{d \varphi}+w\right)+\mu \frac{u \cos \varphi+w \sin \varphi}{R(1+\alpha \sin \varphi)}\right], \\
T_{2} & =K\left[\frac{u \cos \varphi+w \sin \varphi}{R(1+\alpha \sin \varphi)}+\frac{\mu}{a}\left(\frac{d u}{d \varphi}+w\right)\right], \\
M_{1} & =-\frac{D}{a}\left[\frac{1}{a} \frac{d}{d \varphi}+\frac{\mu \cos \varphi}{R(1+\alpha \sin \varphi)}\right]\left(\frac{d w}{d \varphi}-u\right), \\
M_{2} & =-\frac{D}{a}\left[\frac{\cos \varphi}{R(1+\alpha \sin \varphi)}+\frac{\mu}{a} \frac{d}{d \varphi}\right]\left(\frac{d w}{d \varphi}-u\right), \\
N_{1} & =\frac{1}{a} \frac{d M_{1}}{d \varphi}+\frac{\cos \varphi}{R}\left(M_{1}-M_{2}\right),
\end{aligned}
$$

where the stretch stiffness $K=E h /\left(1-\mu^{2}\right)$ and bending stiffness $D=E h^{3} / 12\left(1-\mu^{2}\right), E$ is Young modulus of the nanotorus and $\mu$ is Passion ratio.

The displacement type governing equations for the toroidal shells as follows

$$
\begin{aligned}
{\left[\frac{d}{d \varphi}+\right.} & \left.\frac{(1-\mu) \alpha \cos \varphi}{1+\alpha \sin \varphi}\right]\left(\frac{d u}{d \varphi}+w\right) \\
+ & \alpha\left[\mu \frac{d}{d \varphi}+\frac{(1-\mu) \alpha \cos \varphi}{1+\alpha \sin \varphi}\right] \\
& \times\left(\frac{u \cos \varphi+w \sin \varphi}{1+\alpha \sin \varphi}\right)+\frac{q_{1} a^{2}}{K}=0, \\
\left(\frac{d}{d \varphi}+\right. & \left.\frac{\alpha \cos \varphi}{1+\alpha \sin \varphi}\right) \frac{d}{d \varphi}\left(\frac{d}{d \varphi}+\frac{\alpha \mu \cos \varphi}{1+\alpha \sin \varphi}\right)\left(\frac{d w}{d \varphi}-u\right) \\
+ & \frac{(1-\mu) \alpha}{1+\alpha \sin \varphi}\left(\cos \varphi \frac{d}{d \varphi}-\sin \varphi\right) \\
& \times\left(\frac{d}{d \varphi}+\frac{\alpha \cos \varphi}{1+\alpha \sin \varphi}\right)\left(\frac{d w}{d \varphi}-u\right)+12\left(\frac{a}{h}\right)^{2} \\
& \times\left[\left(1+\frac{\alpha \mu \sin \varphi}{1+\alpha \sin \varphi}\right)\left(\frac{d u}{d \varphi}+w\right)+\alpha\left(\mu+\frac{\alpha \sin \varphi}{1+\alpha \sin \varphi}\right)\right. \\
& \left.\times \frac{u \cos \varphi+w \sin \varphi}{1+\alpha \sin \varphi}\right]=\frac{q_{n} a^{4}}{D},
\end{aligned}
$$

in which, $u$ and $w$ are the displacement components on the middle surface. Note that we have ignored all terms of $\mathrm{O}(h / a)^{2}$ in deriving the first equation of (2), since we are using the Love-Kirchhoff theory of shells, in which all terms of order higher than $\mathrm{O}(h / a)$ are omitted. 
Owing to the complexity of (2), it is very difficult to find an exact solution for a general ratio of $\alpha=a / R$. In the following section, we will propose a closed form solution of a slender toroidal shell where the ratio is very small and can be omitted.

\section{Nanotorus Shell Model and Closed-Form Solution [9]}

As nanotube has been constructed with length-to-diameter ratio of up to $132,000,000: 1$, which is significantly larger than any other material. It means that nanotorus's $\alpha=a / R$ is much smaller than "1", and it means that $\alpha \ll 1$ and $|\sin \varphi| \leq 1$, so that the term $1+\alpha \sin \varphi \approx 1$. It gives a possibility for the simplification and removing its singularity of the toroidal shell equations by ignoring the terms with $\alpha=a / R$ from (2). The toroidal shell is called a slender toroidal shell when $\alpha=a / R$ can be omitted. Although we are not able to solve the toroidal shell with arbitrary $\alpha=a / R$, from engineering point of view the slender toroidal shell is a quite good approximation of general toroidal shells. Mathematically, this simplification can be considered as base of perturbation approach if we take $\alpha=a / R$ as small perturbation parameter.

Applying $\alpha \rightarrow 0$ to (1) and (2), we have equations for the slender toroidal shell as follows:

$$
\begin{gathered}
\frac{d}{d \varphi}\left(\frac{d u}{d \varphi}+w\right)+\frac{q_{1} a^{2}}{K}=0 \\
\frac{d^{3}}{d \varphi^{3}}\left(\frac{d w}{d \varphi}-u\right)+12\left(\frac{a}{h}\right)^{2}\left(\frac{d u}{d \varphi}+w\right)=\frac{q_{n} a^{4}}{D} .
\end{gathered}
$$

This six-order ordinary differential equations have constant coefficient! Its solution will be in closed form. For unknown reason, the equations have never been derived before.

The internal resultant forces and moments are as follows:

$$
\begin{gathered}
T_{1} \approx \frac{K}{a}\left(\frac{d u}{d \varphi}+w\right), \quad T_{2} \approx \frac{\mu K}{a}\left(\frac{d u}{d \varphi}+w\right), \\
M_{1} \approx-\frac{D}{a^{2}} \frac{d}{d \varphi}\left(\frac{d w}{d \varphi}-u\right), \quad M_{2} \approx-\frac{\mu D}{a^{2}} \frac{d}{d \varphi}\left(\frac{d w}{d \varphi}-u\right), \\
N_{1} \approx-\frac{D}{a^{3}} \frac{d^{2}}{d \varphi^{2}}\left(\frac{d w}{d \varphi}-u\right) .
\end{gathered}
$$

Then we have following closed form solution:

$$
\begin{aligned}
\chi(\varphi)= & -2 C_{1}\left(\frac{a}{h}\right)^{2} \frac{a^{2}}{K} \varphi^{3}+C_{2}+C_{3} \varphi+C_{4} \varphi^{2} \\
& +\frac{a^{4}}{D} \int_{\theta}(\varphi-\xi)^{2} q_{n}(\xi) d \xi \\
& +12\left(\frac{a}{h}\right)^{2} \frac{a^{2}}{K} \int_{\theta}(\varphi-\xi)^{3} q_{1}(\xi) d \xi,
\end{aligned}
$$

where $\chi$ is the rotation of normal vector of the middle surface.

We have displacements as follows:

$$
\begin{aligned}
u= & C_{5} \cos \varphi+C_{6} \sin \varphi+\int_{\varphi} p(\xi) \sin (\varphi-\xi) d \xi, \\
w= & \frac{a^{2}}{K}\left[C_{1}-\int_{\varphi} q_{1}(\xi) d \xi\right]+C_{5} \sin \varphi \\
& -C_{6} \cos \varphi-\int_{\varphi} p(\xi) \cos (\varphi-\xi) d \xi,
\end{aligned}
$$

where

$$
\begin{aligned}
p(\varphi)= & -\frac{q_{1} a^{2}}{K}+2 C_{1}\left(\frac{a}{h}\right)^{2} \frac{a^{2}}{K} \varphi^{3}-C_{2}-C_{3} \varphi-C_{4} \varphi^{2} \\
& -\frac{a^{4}}{D} \int_{\varphi}(\varphi-\xi)^{2} q_{n}(\xi) d \xi \\
& -12\left(\frac{a}{h}\right)^{2} \frac{a^{2}}{K} \int_{\varphi}(\varphi-\xi)^{3} q_{1}(\xi) d \xi .
\end{aligned}
$$

The six integration constants $C_{i}(i=1, \ldots, 6)$ can be determined by using boundary conditions.

\section{Closed-Form Solution under Concentrated Loading Condition [9]}

In the case of concentrated loading condition, the above solution can be written in a simpler form. Since now $q_{1}=$ $q_{n}=0$, we have displacement components as follows:

$$
\begin{aligned}
u= & C_{5} \cos \varphi+C_{6} \sin \varphi+2 C_{1}\left(\frac{a}{h}\right)^{2} \frac{a^{2}}{K} \varphi^{3} \\
& -C_{4} \varphi^{2}-C_{3} \varphi-C_{2}, \\
w= & \frac{a^{2}}{K} C_{1}+C_{5} \sin \varphi-C_{6} \cos \varphi \\
& -2 C_{1}\left(\frac{a}{h}\right)^{2} \frac{a^{2}}{K}\left(3 \varphi^{3}+6\right)+C_{3}+2 C_{4} \varphi,
\end{aligned}
$$

and its rotations: $\chi=-2 C_{1}(a / h)^{2}\left(a^{2} / K\right) \varphi^{3}+C_{2}+C_{3} \varphi+C_{4} \varphi^{2}$. The internal forces and moments are given by $T_{1}=a C_{1}$, $M_{1}=-\left(D / a^{2}\right)\left[-6 C_{1}(a / h)^{2}\left(a^{2} / K\right) \varphi^{2}+C_{3}+2 C_{4} \varphi\right], N_{1}=$ $-\left(D / a^{3}\right)\left[-12 C_{1}(a / h)^{2}\left(a^{2} / K\right) \varphi+2 C_{4}\right]$.

As an example, we can apply the above solution (9) to Figure 5; the separation displacement deformation of the nanotorus can be obtained as $\delta_{\text {Sun }}=\left(\pi^{2} / 6\right)\left(P a^{3} / R D\right)=$ $1.644934\left(\mathrm{~Pa}^{3} / R D\right)$, where bending stiffness $D=E h^{3} /[12(1-$ $\left.\left.\mu^{2}\right)\right], E$ is Young modulus of the nanotorus. It is a very simple solution, which can be used for verification of both experimental results and atomistic simulation in the future.

\section{Nanotorus Embedded in Elastic Medium (Winkler Model)}

When the nanotube or nanotorus embedded in composite materials, the nanotorus-matric interaction can be viewed 
as a nanotorus buried in a elastic foundation, which can be described as a Winkler model. There are some investigations on nanotube buried in the elastic medium $[7,8]$. Unfortunately, there is no any paper has considered the case of nanotorus embedded in elastic medium. As a natural extension of this work, we will present closedform solution of the nanotorus embedded in Winkler elastic medium.

In Winkler model, the loading distribution in normal direction of the nanotorus will be in the form of $q_{n}=q-k w$, where $k$ is the Winkler constant, which is determined by the material properties of the elastic matrix and curvature of the nanotorus. Here, the dependency of the constant $k$ on the curvature can be neglected as a second-order effect.

After introducing the Winkler model, the governing equation of the nanotorus embedded in elastic medium take the following form:

$$
\begin{gathered}
\frac{d}{d \varphi}\left(\frac{d u}{d \varphi}+w\right)=0 \\
\frac{d^{3}}{d \varphi^{3}}\left(\frac{d w}{d \varphi}-u\right)+12\left(\frac{a}{h}\right)^{2}\left(\frac{d u}{d \varphi}+w\right)+k \frac{a^{4}}{D} w=\frac{q a^{4}}{D} .
\end{gathered}
$$

in which, team $q_{1} a^{2} / K$ has to be removed in the first equation of (10) since we are not considering the friction between nanotorus and matrix. Then we have

$$
\begin{gathered}
w=C_{1}-\left(\frac{d u}{d \varphi}\right), \\
u=\sum_{i=3}^{6} C_{i} e^{\lambda_{i} \varphi}+\frac{D}{k a^{4}}\left[-\frac{q a^{4}}{D} \varphi+12\left(\frac{a}{h}\right)^{2} C_{1} \varphi+k \frac{a^{4}}{D} C_{1} \varphi+C_{2}\right], \\
\lambda_{i}= \pm \sqrt{\frac{-1 \pm \sqrt{1-4\left(k a^{4} / D\right)}}{2}}
\end{gathered}
$$

\section{Vibration of Slender Toroidal Shells}

The advantage of using the displacement type equations is that we are able to study the deformation as well as the vibration of the shells. For literal vibration, the vibration in the direction of $u$ can be ignored due to its relatively small value. Then equation becomes, by deleting the inertial term $\left(\rho h a^{2} / K\right) \ddot{u}$,

$$
\begin{gathered}
\frac{\partial}{\partial \varphi}\left(\frac{\partial u}{\partial \varphi}+w\right)=0 \\
\frac{\partial^{3}}{\partial \varphi^{3}}\left(\frac{\partial w}{\partial \varphi}-u\right)+12\left(\frac{a}{h}\right)^{2}\left(\frac{\partial u}{\partial \varphi}+w\right)+\frac{\rho h a^{4}}{D} \ddot{w}=0
\end{gathered}
$$

where $\rho$ is the density of the shell material and the partial derivative with respect to time $\dot{u}=\partial u / \partial t$. The solution is given by

$$
\begin{aligned}
u(\varphi, t)= & {\left[b_{3} e^{\beta_{1} \varphi}+b_{4} e^{\beta_{2} \varphi}+b_{5} e^{\beta_{3} \varphi}+b_{6} e^{\beta_{4} \varphi}\right.} \\
& \left.\quad-\frac{1}{\omega^{2}} \frac{D}{\rho h a^{4}}\left(\frac{12 a^{2}}{h^{2}} b_{1} \varphi+b_{2}\right)\right] \\
& \times\left(B_{1} \cos \omega t+B_{2} \sin \omega t\right) \\
w= & b_{1}-\left[b_{3} \beta e_{1}^{\beta_{1} \varphi}+b_{4} \beta_{2} e^{\beta_{2} \varphi}+b_{5} \beta_{3} e^{\beta_{3} \varphi}+b_{6} \beta_{4} e^{\beta_{4} \varphi}\right. \\
& \left.\quad-\frac{12}{\omega^{2}} \frac{D}{\rho h^{3} a^{2}} b_{1}\right]\left(B_{1} \cos \omega t+B_{2} \sin \omega t\right)
\end{aligned}
$$

where $b_{i}(i=1,2,3,4,5,6)$ are integration constants and $\beta_{i}=$ $\pm \sqrt{-1 \pm \sqrt{\left(1+4\left(\rho h a^{4} / D\right) \omega^{2}\right)} / 2}$.

With the solution of the free vibration problem, any forced vibration can be derived by using the superposition principal of vibration modes. This is a standard operation which has no any difficult.

\section{Buckling of Nanotorus}

General speaking, the buckling of toroidal shells is a very difficult problem, and no analytical solution has been obtained. By taking advantage of the displacement form of the governing equations for toroidal shells, we can find its analytical solution of the buckling problem.

The buckling equation of the slender toroidal shell is as follows:

$$
\begin{gathered}
\frac{d}{d \varphi}\left(\frac{d u}{d \varphi}+w\right)=0 \\
\frac{d^{3}}{d \varphi^{3}}\left(\frac{d w}{d \varphi}-u\right)+12\left(\frac{a}{h}\right)^{2}\left(\frac{d u}{d \varphi}+w\right) \\
+\frac{T_{1}^{*}}{a} \frac{d}{d \varphi}\left(\frac{d w}{d \varphi}-u\right)+T_{2}^{*} \frac{\cos \varphi}{R}\left(\frac{d w}{d \varphi}-u\right)=0
\end{gathered}
$$

where $T_{1}^{*}, T_{2}^{*}$ are the internal forces for the zero-moment state of the shells, which satisfies the membrane balance equation $\left(T_{1}^{*} / R_{1}\right)+\left(T_{2}^{*} / R_{2}\right)=q_{n}$. The first equation of $(15)$ becomes $(d u / d \varphi)+w=-(1 / 12)(a / h)^{2} d_{1}$, in which $d_{1}$ is an integration constant. The second equation of (15) becomes

$$
\frac{d^{3} \chi}{d \varphi^{3}}+\frac{T_{1}^{*}}{a} \frac{d \chi}{d \varphi}+T_{2}^{*} \frac{\cos \varphi}{R} \chi=d_{1} .
$$

This is the buckling equation for slender toroidal shells, where rotation of normal vector of the nanotorus $\chi=$ $(d w / d \varphi)-u$. 
It is worth noting that the strain $\varepsilon_{1}=(1 / a)((d u / d \varphi)+$ $w)=-(1 / 12)\left(a / h^{2}\right) d_{1}$ is a constant and is independent of $q_{n}$; this is a special feature of nanotorus. For a nanotorus undergoing a distributed compressive loading, $T_{1}^{*}=a q_{n}$, $T_{2}^{*}=(1 / 2) a q_{n},(16)$ becomes

$$
\frac{d^{3} \chi}{d \varphi^{3}}+q_{n} \frac{d \chi}{d \varphi}+\underline{\frac{1}{2}\left(\frac{a}{R} \cos \varphi\right) \chi}=d_{1} .
$$

The underlined term can be ignored due to the fact that the ratio $\alpha=a / R$ is very small. Then the buckling equation for a slender toroidal shell becomes

$$
\frac{d}{d \varphi}\left(\frac{d^{2} \chi}{d \varphi^{2}}+q_{n} \chi\right)=d_{1} \quad \text { or } \quad \frac{d^{2} \chi}{d \varphi^{2}}+q_{n} \chi=d_{1} \varphi+d_{2} .
$$

Its solution can be easily found by the theory of ordinary differential equations

$$
\chi=d_{3} \cos \sqrt{q_{n}} \varphi+d_{4} \sin \sqrt{q_{n}} \varphi+\frac{1}{q_{n}}\left(d_{1} \varphi+d_{2}\right),
$$

where $d_{i}$ are integration constants. The displacements are as follows:

$$
\begin{aligned}
u= & d_{5} \cos \varphi+d_{6} \sin \varphi-\frac{1}{1-q_{n}} d_{3} \cos \sqrt{q_{n}} \varphi \\
& -\frac{1}{1+q_{n}} d_{4} \sin \sqrt{q_{n}} \varphi-\frac{1}{q_{n}}\left(d_{1} \varphi+d_{2}\right), \\
w= & {\left[\frac{1}{q_{n}}-\frac{1}{12}\left(\frac{a}{h}\right)^{2}\right] d_{1}+d_{5} \sin \varphi-d_{6} \cos \varphi } \\
& -d_{3} \frac{\sqrt{q_{n}}}{1-q_{n}} \sin \sqrt{q_{n}} \varphi+d_{4} \frac{\sqrt{q_{n}}}{1+q_{n}} \cos \sqrt{q_{n}} \varphi .
\end{aligned}
$$

The internal forces and moments are given by

$$
\begin{aligned}
& T_{1}=\frac{K}{a}\left(\frac{d u}{d \varphi}+w\right)=-\frac{1}{12} \frac{a}{h^{2}} d_{1}, \\
& M_{1}=-\frac{D}{a^{2}} \frac{d \chi}{d \varphi} \\
& =-\frac{\sqrt{q_{n}} D}{a^{2}}\left[-d_{3} \sqrt{q_{n}} \sin \sqrt{q_{n}} \varphi+d_{4} \sqrt{q_{n}} \cos \sqrt{q_{n}} \varphi+\frac{1}{q_{n}} d_{1}\right], \\
& N_{1}=-\frac{D}{a^{2}} \frac{d^{2} \chi}{d \varphi^{2}}=\frac{q_{n} D}{a^{2}}\left[d_{3} \cos \sqrt{q_{n}} \varphi+d_{4} \sin \sqrt{q_{n}} \varphi\right] .
\end{aligned}
$$

Using proper boundary conditions, the critical value of $q_{n}$ can be obtained as an eigenvalue of the problem.

\section{Conclusion}

In the light of continuum model and the factor of very large length-to-diameter ratio of nanotorus, we successfully formulated the deformation, vibration, and buckling of the nanotorus embedded in an elastic medium by using toroidal shell model. The mathematical complicated equations of the general nanotorus have been reduced to simple differential equations with constant coefficients, which leads to a successful finding of closed-form solution of nanotorus. An example has been demonstrated for the case of concentrated loading condition. All solutions obtained here will be very useful for the future designing nanotori devices.

\section{Acknowledgments}

This paper was supported by South Africa National Research Foundation. This support is gratefully acknowledged. The author would like to express his thankfulness to the constructive comments and insightful suggestions given by three anonymous referees.

\section{References}

[1] S. Iijima, "Helical microtubules of graphitic carbon," Nature, vol. 354, no. 6348, pp. 56-58, 1991.

[2] B. Sun and X. M. H. Huang, "Mechanical nano-resonators at ultra-high frequency and their potential applications," South African Journal of Science, vol. 104, no. 5-6, pp. 169-171, 2008.

[3] B. I. Yakobson, C. J. Brabec, and J. Bernholc, "Nanomechanics of carbon tubes: instabilities beyond linear response," Physical Review Letters, vol. 76, p. 2511, 1996.

[4] O. Hod, E. Rabani, and R. Baer, "Carbon nanotube closed-ring structures," Physical Review B, vol. 67, no. 19, Article ID 195408, 7 pages, 2003.

[5] M. Huhtala, A. Kuronen, and K. Kaski, "Carbon nanotube structures: molecular dynamics simulation at realistic limit," Computer Physics Communications, vol. 146, no. 1, pp. 30-37, 2002.

[6] T. Çağin, G. Gao, and W. A. Goddard III, "Computational studies on mechanical properties of carbon nanotori," Turkish Journal of Physics, vol. 30, no. 4, pp. 221-229, 2006.

[7] O. Lourie, D. M. Cox, and H. D. Wagner, "Buckling and collapse of embedded carbon nanotubes," Physical Review Letters, vol. 81, no. 8, pp. 1638-1641, 1998.

[8] C. Q. Ru, "Axially compressed buckling of a doublewalled carbon nanotube embedded in an elastic medium," Journal of the Mechanics and Physics of Solids, vol. 49, no. 6, pp. 1265$1279,2001$.

[9] B. Sun, "Closed form solution of axisymmetric slender elastic toroidal shells," ASCE Journal of Engineering Mechanics. In press. 

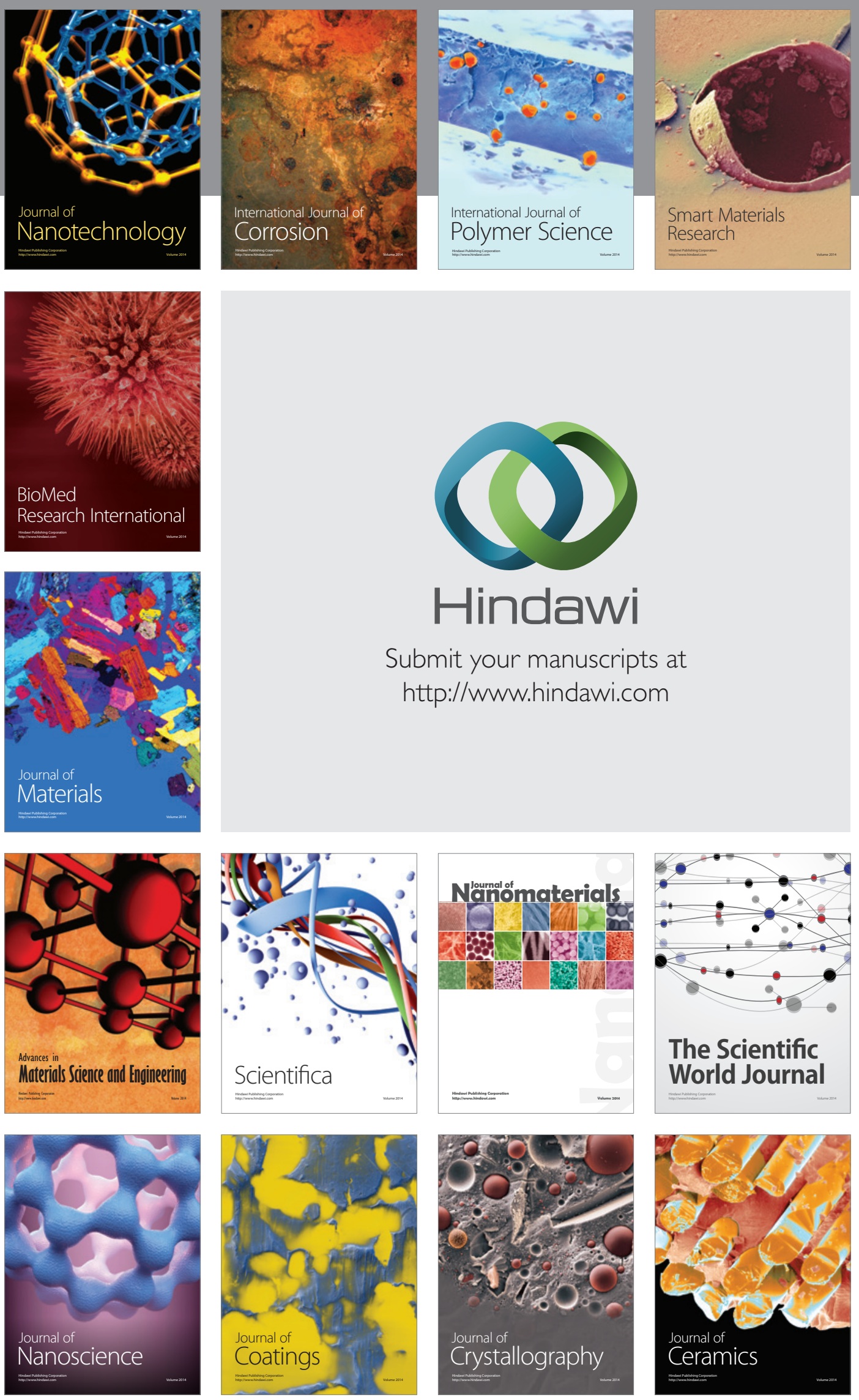

The Scientific World Journal

Submit your manuscripts at

http://www.hindawi.com

\section{World Journal}

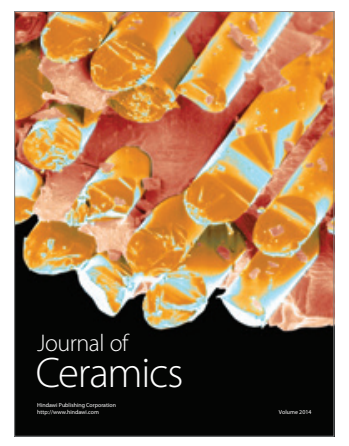

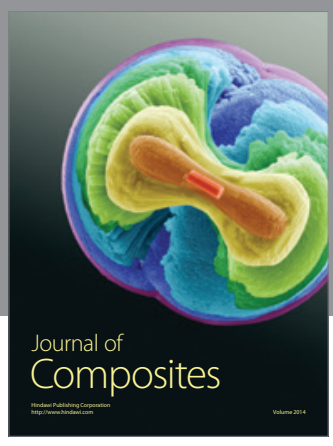
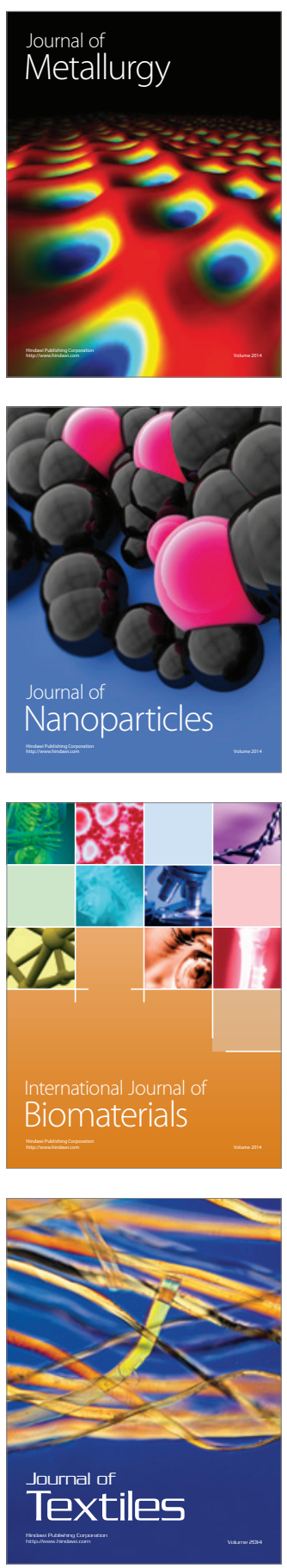